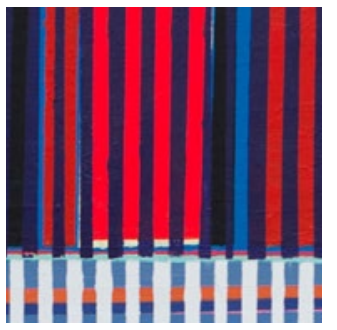

\title{
Identidades comunitarias y políticas indigenistas
}

\author{
COMMUNITY IDENTITIES AND INDIGENIST POLICIES
}

\section{Daniel Gutiérrez Martínez}

El Colegio Mexiquense, A. C. dgutierrezcolmex@yahoo.fr

Recibido: 21/04/2017 - Aceptado 28/08/2017

\section{RESUMEN}

Diferentes respuestas han sido dadas para explicar el proceso identitario que se encierra en la relación entre las políticas públicas y comunidades. Uno de ellos es el de la aculturación cara a Aguirre Beltrán (2013, [1957]). Por otro lado, el sincretismo también ha fungido como modelo explicativo en la relación denominada identidad indígena y políticas públicas del Estado-Nacional.

El debate de las identidades móviles y flexibles, sin duda tiene raíces desde los años setenta del siglo pasado, no obstante es a fines del siglo pasado que empezó a tomar fuerza en el ámbito de la política y tomó auge y renovación a principios del siglo XXI. Desde la antropología estructural con Lévi-Strauss hasta la antropología simbólica de Cliffort Geertz, la noción de identidades ha evolucionado mucho y aportado enormemente a la comprensión de la conformación de los significados sociales, sin embargo, consideramos que todavía no se ha completado una teoría total del fenómeno de las identidades. Más allá de las identidades móviles y flexibles, están los sentidos/ sentimientos de pertenencia que aglutinan y distinguen. Este será la propuesta específica de esta reflexión.

Palabras clave: Sentimiento de pertenencia, umbral de identidades, hibridación, resignificación, indigenismos contemporáneos.

\section{ABSTRACT:}

Different answers have being given to explain the identity process in the relationship between public policies and communities. One of them is acculturation, so dear to Aguirre Beltrán (2013, [1957]) and, on the other hand, syncretism has also being used as a model to explain the so called indigenist identity-public policies relationship.

The debate on mobile and flexible identities no doubt has its roots in the 70's but by the end of the last Century it began to gain its place in the political area to gather further energy at the beginning of the XXI Century. From the structural anthropology with Lévi-Strauss to the sym- 
bolic anthropology of Clifford Geertz, the identity notion has evolved a great deal and provided an enormous input to the understanding of how social meanings are formed. Nevertheless we consider that a total theory on identities has yet to be completed. Not only mobile and flexible identities but we have to include senses, feelings of belonging that agglutinate and distinguish regarding the others. This will by the specific proposal of this article.

Key words: feeling of belonging; identities threshold; hybridization; resignification; contemporary indigenisms.

\section{Introducción}

El análisis y comprensión de las identidades que se procesan día a día en las comunidades, en los pueblos y localidades nativas del mundo (cualquiera sea el grupo de referencia), han sido motivo de debate y grandes controversias en el ámbito académico e instancias políticas. ${ }^{1}$ La mayoría se ha remitido a reflexionar desde la mirada institucional del Estado, en las formas de constitución de la identidad llamada "indígena", tendiendo a enfocarse en las dimensiones de lo "popular" y étnico, oscilando en presupuestos folcloristas, exóticos, esencialistas, a veces insertos en el clásico debate de la aculturación, más aún el de las identidades móviles y dinámicas.

Paradójicamente, desde que se avanzó teóricamente con la idea de que las identidades son flexibles y procesos en constante cambio, han aumentado los reduccionismos analíticos y esquematizaciones identitarias en la dimensión política, especialmente en torno a los que se han denominado "pueblos indígenas". Ahora resulta que hay "indígenas" con identidades móviles, movibles y flexibles, pero sin abandonar los insumos teóricos esencialistas de antaño que los determinaron; pues siguen siendo considerados indígenas (lo que esto refiera en términos identitarios), aunque sea con connotación positiva, reivindicativa o valorativa. Dicha noción, de todos modos, trae consigo una carga valorativa histórica de colonialismo, que pretende definir a grupos humanos en particular desde hace, al menos, más de quinientos años.

Es recurrente ahora escuchar a cientistas sociales y políticos decir que visto que los miembros de las comunidades "indígenas" ya se asumen como tales, incluso para hacer frente al gobierno (indigenismos revolucionarios), es irrelevante ahora que se cuestione la validez o pertinencia conceptuales del vocablo. El hecho de que las comunidades nativas y locales se autodenominen "indígenas", no significa que hayan legitimado a cabalidad dicho vocablo, cargado de historia colonialista, ${ }^{2}$ más bien nos da cuenta de la

1. Entendemos pueblos y comunidades nativas a todos aquellos grupos oriundos de un lugar y/ o tiempo específicoscon sentimientos de pertenencia simbólicos y/ o físicos y con raíces históricas milenarias. Nativo: relativo a nacer en el lugar. Por lo tanto también se podrían entender como pueblos locales o simplemente localidades. Lo anterior remite a la consciencia de base, que histórica, geográfica, culturalmente un pueblo pueda tener de sí mismo, la cual termine por vehicular y participar en procesos identitarios. Pueblo remite al sentimiento de pertenencia a un grupo, a una serie de valores, principios y creencias que producen sentidos de la acción colectivos. Por lo general los debates y controversias a los que hacemos referencia alrededor de las identidades que se procesan día a día en las comunidades y pueblos, en el ámbito académico e instancias políticas tienen que ver con lo que el Estado llama pueblos "indígenas o políticas indigenistas”. Si bien indígenas remite etimológicamente a los lugareños, es cierto que a partir del siglo XVII pasa a tener connotaciones colonialistas. Por ello, más que pueblos indios, indígenas, u originarios, preferimos usar los términos de pueblos comunitarios, pueblos nativos o nacionales, con el fin de ubicar a todos aquellos grupos con sentimientos de pertenencia colectivos, más allá del Estado-nacional, fundados en la historia de larga duración y compartiendo valores y creencias más allá de los modernos que promueven los Estados-Nacionales, estados republicanos o instituciones seculares modernas.

2. Algunos autores, hablaron de colonialismo interno a los efectos excluyentes y marginalizantes que producen políticas de Estado sobre las poblaciones concernidas. Nosotros lo llamamos aquí Conquista dóxica por 
violencia simbólica (Bourdieu, 1994) que se ha ejercido sobre las poblaciones con este término al punto de usarlo como insumo identitario, sobre todo frente al Estado.¿Cómo valorar la auto-denominación "indígenas" por parte de comunidades nativas, localidades o barrios cuando se sabe que la sola auto-denominación otorga derechos culturales, económicos, políticos frente a las instituciones de Estado; esto incluye desde apoyos, becas, premios de poesía en lenguas maternas, etc.? En algunos sitios se presta a la complacencia o anuencia del líder del pueblo para avalar a una persona o grupo como miembros de la comunidad. Ni se diga en las universidades, las cuales exigen cuasi "apelaciones de origen controlado" para otorgarles becas, patrimonios y patrocinios. Eso sí es muy importante, que los beneficiarios sigan promoviendo temáticas, paisajes, proyectos con insumos "indigenistas" para supuestamente conservar y preservar sus culturas (lo que esto quiera decir).

Ante todo son grupos y comunidades como cualquiera, compartiendo intersubjetividades comunes y colectivas pasadas y presentes que conforman día a día su existencia y su constante variabilidad, más allá de lo que se le pueda adjudicar al adjetivo identitario, con los juicios de valor positivos y/ o idílicos que contengan. Son supuestos adjetivados que poco ayudan a entender procesos identitarios y con-formaciones de culturas locales (cualquiera sean éstas), desde el ámbito analítico y científico. Hay quienes dan por sentada esta fórmula como realidad, para hacer distinción entre un tipo de identidad (la de los indígenas) y las otras (la de los ciudadanos modernos: criollos, mestizos, urbanos, etc.) y con ello se establecen grupos especiales con supuestas actitudes particulares. El concepto de "indígena", más bien permite identificar las hegemónicas relaciones asimétricas de poder entre instituciones y funcionarios de Estado con las poblaciones culturales y pueblos nativos de larga tradición. ${ }^{3}$

Lo importante es dar cuenta de los impasses teóricos cuando las identidades, sobre todo las llamadas "indígenas", se analizan desde aspectos materialistas y exteriorizantes (los de religión, etnia, familia, sexo, edad, principalmente) para determinar comportamientos y sentidos de la acción, donde las identidades se ven supeditadas teóricamente al análisis de los apellidos que se les imputan (identidades profesionales, nacionales, judías, católicas, blancas, afro, etc.). El problema con ello tiene que ver con los idilios, folclorismos o esencialismos, que se generan y que terminan por definir el curso de las políticas de Estado hacia ciertas poblaciones, que a la postre contribuyen a estigmatizaciones identitarias de largo alcance, contribuyendo a su vez a la reproducción de las desigualdades sociales y económicas que se presume erradicar. Lo anterior nos permite dar cuenta, desde el análisis de la identidad, de la manera en la cual miembros de pueblos nacionales (no sólo en México, sino en cualquier parte del mundo) hacen uso de diferentes recursos identitarios para adaptarse al entorno que los envuelve, impuesto desde el exterior (resignificación/ hibridación).

En suma, esta reflexión busca: I) por un lado, dar cuenta que cualquiera sean los procesos de identidad de referencia, tienen como base primera y fundamental los sentidos/ sentimientos de pertenencia generados colectivamente; estos unen y distinguen

el carácter interferente identitario que contienen dichas políticas a lo largo de al menos quinientos años sobre pueblos comunitarios, locales o nativos. Como ahondaremos más adelante, re-significación o hibridación son los efectos que tienen sobre los procesos identitarios las políticas públicas de Estado, las cuales nos revelan, las relaciones de poder hegemónicas, o relaciones de fuerza que se producen entre Estado y poblaciones.

3. Lo anterior se aplica no sólo para la noción de "indígena", sino para la del civilizado, el criollo, el mestizo, el urbano, el ilustrado, etc. No son más que categorías construidas para denominar lo que se quiere hacer valer como normal, y lo que es "anormal" según la ideología predominante. 
a los grupos humanos; II)se trata también de dar cuenta del impacto de las políticas públicas estatales implantadas en las últimas décadas sobre los procesos identitarios conformados en poblaciones que el Estado-nacional 1lama "indígenas"; III) finalmente, se busca dar cuenta de las acciones y estrategias identitarias de hibridación y re-significación (ejemplificados con estudios de caso), que integrantes de pueblos y comunidades nativas en México han llevado a cabo, en reacción a las implementaciones de políticas indigenistas estatales.

\section{I) Perspectivas heurísticas y prospección teórica}

Como toda construcción académica, la identidad, es un término en constante actualización, reflejando la movilidad de épocas, de pensamientos, de concepciones, de posturas. El enfoque que se plantea de la identidad ha variado según la escuela donde los teóricos de las identidades se posicionan, desde la época de correspondencia. Aquí es donde la discusión y el debate empiezan. Por lo general, las diferentes conceptuaciones de la(s) identidad(es) comienzan por cuestionar su definición como una sustancia, un atributo inmutable del individuo y de las colectividades. Hay quienes ponen en duda completamente el término de identidades, remitiéndonos a la noción de identificaciones o a un estar-juntos antropológico (Maffesoli 2007). ${ }^{4}$ Se ha hecho referencia a modos de adhesión y constitución de identificaciones movibles, efímeras y flexibles. No se ha trabajado mucho desde la dimensión de las identidades como procesos de conformaciones de significados sociales, colectivamente constituidos con y en el entorno, desde los aspectos interaccionales constantes de subjetividades y emocionalidades colectivas,

30 y a partir de los sentimientos/ sentidos de pertenencia compartidos (emociones colectivas). Las instituciones de socialización e identitarias como la familia, los partidos políticos, las iglesias, los Estados, etc., fungen como compensación conceptual entre el papel que tienen estas instituciones de socialización y el papel que desempeñan los aspectos colectivos personales y estructurales en estos procesos: se trata de resaltar la interacción colectiva de emociones conformando sentidos/sentimientos de pertenencia breves y perennes. La interacción con el otro, en tanto mediación con el entorno (otredad) produce identidades, las cuales tienen determinantes en los marcos mental y subjetivo, siendo insumos de experiencias singulares, insertadas en instituciones o grupos de pertenencia que canalizan la acción y proporcionan sentidos y legitimaciones simbólicas. Son elaboraciones colectivas que se conforman y se actualizan sin cesar en las interacciones entre personas, grupos con sus ideologías y creencias confluyendo con la lógica interna del mismo proceso. Es un enfoque relacional e interaccional, que no privilegia las acciones o actores, sino la relación e interacción en sí mismas. Metodológicamente la identidad es el crisol donde diversidad e intersubjetividades entre grupos humanos se conectan, estimulados por el entorno, que son compartidos y terminan por con-formar (en conjunto) culturas una y otra vez.

\section{Enfoques heurísticos para el estudio de las identidades}

Toda conformación identitaria genera un discurso, una narrativa, que se ven atravesados por mecanismos culturales, evaluaciones morales y construcciones políticas y sociales.

4. Toda la obra de Michel Maffesoli reflexiona en torno a las identificaciones y los estar/ser-juntos antropológicos, ver al respecto Maffesoli 2007, 2005, 2004. 
Dicho proceso está en constante actualización/adaptación, re-significación donde según el momento hay dinámicas prevaleciendo procesos de unión/ vinculación; separación/ distinción, los dos paralelamente. Esta característica de unir y distinguir es el cimiento de toda cultura y toda sociedad. El proceso identitario contiene factores de pertenencia básicas: los sentimientos y los sentidos.

Los sentimientos de pertenencia (identitarios) refieren a procesos continuos y contenidos en la memoria colectiva de adscripciones grupales con objetivos y significados sociales. Son procesos de la larga duración (en términos braudelianos) que tienden a ser estables en el tiempo-espacio (religiones, etnicidad, nacionalismos). Son dinámicas que resultan en procesos de homologación, y particularmente de unión, lazo, mismidad. Es lo que aglutina: las llamadas identidades tradicionales e institucionales.

Los sentidos de pertenencia (identificaciones) hacen referencia a acciones de interpretación y/o representación vinculadas a la emotividad colectiva, es decir: sentimientos y pensamientos en conexión impulsados por factores culturales (medio ambiente y los entornos natural, social, geográfico, ambiental, poblacional, emociones colectivas). La conformación identitaria del grupo o personas contiene lógicas de diferenciación, de separación, de distinción vinculadas específicamente con aspectos de poca duración en la experiencia vivida: son contemporáneas e instituyentes. Las identificaciones (sentidos de pertenencia) son adscripciones o apropiaciones fragmentadas que coexisten de manera momentánea y espontánea a la vez, en el proceso interactivo. Las identificaciones o sentidos de pertenencia corresponden a procesos más flexibles y movibles que los sentimientos de pertenencia, que también se ven ligados a la cuestión histórica y de la memoria, empero aquí describe al sujeto fragmentado, itinerante, más allá de la tradición, y alimenta a la con-formación de procesos híbridos o de resignificación de identidad, por lo mismo que generan sentidos de pertenencia (significados y objetivos sociales compartidos del momento). Son cristalizaciones de adscripciones culturales, es decir, entidades que contienen rasgos comunes compartidos por una comunidad en tiempo y espacio. A través de la cultura nos conectan con lo primero y fundamental (del griego arché) de las inter-subjetividades colectivas. Estas dos características del proceso de identidad no define determinismos, sino estructuraciones simbólicas en la larga duración: imaginarios. Sentimientos y sentidos de pertenencia son interdependientes y actúan al mismo tiempo en diferentes dimensiones. Esta abstracción, nos sitúa más en un nivel local que universalista o determinista del concepto de identidades, pues describe el proceso que va de los sentimientos a los sentidos de pertenencia al aludir primeramente sentimientos de pertenencia de un miembro de una comunidad con su familia, después con su comunidad de origen, posteriormente con su grupo étnico, finalmente con su nacionalidad, donde estas últimas pueden ser más endebles que las demás. ${ }^{5}$ Lo mismo aplica para un "mestizo" urbano o cualquier persona o grupo que pudiera llegar a sentirse identificado, primero con su familia, después con su barrio, ciudad o

\footnotetext{
5. Si tomamos el caso de los procesos identitarios que se enarbolan lejos de las comunidades de origen, por ejemplo, el caso de hijos de otomíes de México, que nacieron y viven en USA, notaremos que primero se sienten identificados con su familia, en este caso también con la comunidad de donde vienen sus progenitores (por ejemplo del pueblo de Guerrero en el Municipio de Anaya, Hidalgo, México), luego se sentirán identitarios con la nación otomí en general, después con la nacionalidad mexicana (sentimientos de pertenencia), pero a la vez les generará también todo el sentido de pertenencia el hecho de identificarse como estadounidenses porque nacieron ahí y tienen la nacionalidad de ese país. También quizás se sentirán californianos, a lo mejor finalmente de San Francisco, ciudad donde nacieron. No hablarán quizás ni el otomí, y poco el español, pero el sentir identitario les genera sentido (significación) en EUA, pues ello los hace diferentes de los mexicanos en general, y de los demás grupos culturales viviendo en EUA, que es un país pluricultural.
} 
región, y después con el territorio nacional. Benedict Anderson habla de comunidades imaginadas "porque aún los miembros de la nación más pequeña no conocerán jamás a la mayoría de sus compatriotas, no los verán ni oirán siquiera hablar de ellos, pero en la mente de cada uno vive la imagen de su comunión" (Anderson, 1993, p 212).

Sin desdeñar los cuestionamientos en torno a las teorías de las identidades, éstas fungen como herramientas heurísticas para el análisis de las conformaciones culturales y sociales en diferentes niveles de análisis (véase micro, macro o meso) asociados a distinciones y aglutinaciones de pertenencia, así como para vincularlos con procesos simbólicos más concretos como las cosmovisiones, véase sistemas compartidos de creencias (infra) y acciones. Pensemos las identidades como procesos, haciendo con ello, el uso de la rejilla de lectura denominada "umbrales de identidad", para estudiar las cosmovisiones estructuralmente compartidas por un grupo, y ubicarse en el nivel de análisis identitario que se desea (micro-meso-macro).

\section{Umbrales de identidades}

Para analizar la con-formación de identidades (sentidos/ sentimientos de pertenencia) a lo largo del tiempo hay que situarnos desde los diferenciales epocales y espaciales en los que se han dado las relaciones e interacciones. Esto refuerza y diversifica lo que llamaremos umbrales. Significa por un lado las etapas, épocas, espacios temporales concatenados en la larga duración que nos permitirán teóricamente analizar procesosfuncionamientos de identidades en el presente, pues es a través de las fijaciones históricas de la memoria colectiva (sentimientos de pertenencia), y su reactivaciónactualización en la interacción cotidiana (sentidos de pertenencia) que se conforman los discursos de identidad. La noción de umbrales también significa conjunciones de procesos identitarios en el presente, diferenciados espacialmente. Tiempo-pasado y espacio-presente se ven reflejados en la noción de umbrales. Son procesos de pertenencia en el tiempo designando precisamente espíritus del tiempo en interacción, es decir, atmósferas, cosmovisiones específicas de cada época y espacios que se van concatenando e interconectando en momentos históricos y lugares geográficos en el proceso de constitución presente de un fenómeno identitario. Se trata de pensar aquellos ambientes que se integran a la realidad social que corresponden a tiempos sociológicos (atmósferas), donde el espíritu del tiempo en cuestión se puede encontrar en cualquier momento del calendario de época. La propuesta metodológica de los umbrales refiere a tipos ideales weberianos con el fin de establecer puntos de arranque para la reflexión del tema planteado, permitiéndonos esquematizar acciones, reacciones, y estrategias observadas. El tipo ideal weberiano puede verse así como un recurso metodológico que plantea un compromiso entre las exigencias de la exactitud histórica y la precisión teórica. En esta reflexión tanto la noción de umbrales, como las de holismo/individualismo, como identidades hibridas y resignificación, son tipos ideales que nos permiten metodológicamente situarnos en nuestro tema de análisis.

\section{II) Identidades comunitarias e indigenismos políticos: sentimientos de pertenencia}

Con los umbrales, podemos manejar herramientas de comprensión del impacto que puedan tener las políticas públicas estatales implantadas en las comunidades (en este caso indigenistas), sobre los procesos identitarios conformados en poblaciones llama- 
das por el Estado- de "indígenas". El punto de vista teórico estructural de los procesos identitarios puede visibilizar inercias humanas a lo largo de la historia. Los umbrales identitarios, nos permiten justamente visibilizar dichas inercias (que son procesos por antonomasia), y hacen visibles las relaciones de fuerza, las predominancias, los determinismos que se expresan en épocas y lugares dados que terminan por conformar identidad. Dumont, Durkheim y Lévi-Strauss, para no citar más que algunos autores emblemáticos, hablan conceptualmente de la interactividad humana en términos de dualidades espacio-tiempo. ${ }^{6}$ El primero lo hace a través de los conceptos de "holismo e individualismo", el segundo en términos de "sociedades orgánicas y mecánicas", y el tercero con nociones de "pensamiento concreto y/ o salvaje, y pensamiento abstracto (universalismo y particularismo)". Para nuestro interés, es en la relación existente entre políticas públicas estatales y pueblos comunitarios que encontramos inscritos la tensión entre estos dos procesos identitarios espacio-temporales, mezclados unos con otros, predominando uno más que otro, según el umbral de referencia.

Solo refiriéndonos al holismo de Louis Dumont, representa una forma de organización social que implica que la persona se define en función del grupo al que pertenece (familia, comunidad, casta o estamento), mientras que el individualismo se caracteriza por resaltar la importancia del mérito de cada individuo para su inserción social: "desde este punto de vista una primera constatación se impone: el individuo es un valor, o más bien forma parte de una configuración de ciertos valores sui generis" (Dumont, 1966). Es la recurrente reflexión sobre la existencia de la dualidad de sociedades en el interior de los grupos humanos, donde según el momento y el tiempo una dinámica societal pesará más que la otra. El enfoque analítico del núcleo duro entrañable a (López Austin, 1999) sería más contemporáneo y heurístico para hablar de estas formas duales de procesos identitarios o cosmovisiones duales, particularmente si se desea vislumbrar las transmisiones de valores de pertenencia en la larga duración (cosmovisiones). El cuadro I remite a cada una de estas nociones.

6. Lo anterior no excluye pensar en términos de triadismo. Maffesoli plantea que "es indudable que la hipótesis del «sentimiento compartido» obliga a repensar el papel del Tercero, es decir, de lo plural en la estructuración societal. (...) La metáfora del triadismo permite poner de manifiesto la paradoja, (...) la pluralidad constitutiva de este neotribalismo contemporáneo". (Maffesoli op., cit.: 192).Véase igualmente (Freund 1983), Cf. asimismo, (Dumezil 1941) y (Durand 1980). Sobre el triadismo a partir de una visión simbolista, Cf. (Durand 1984). 
CUADRO I. UMBRALES (COSMOVISIONES)

NÚCLEOS DUROS IDENTITARIOS

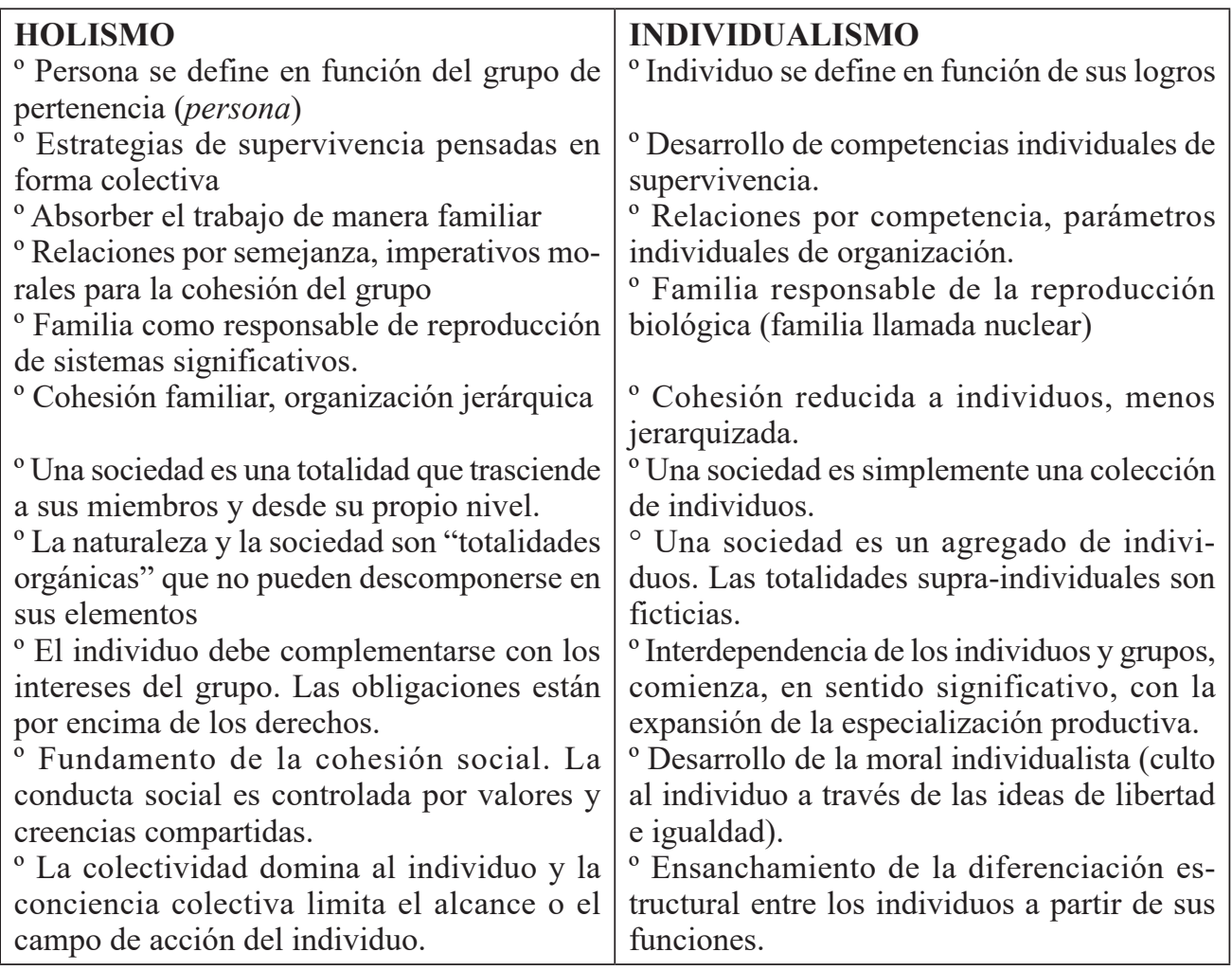

Fuente: Elaboración propia a partir de Dumont (1966)

En todas las sociedades las dos cosmovisiones o núcleos duros perviven y se manifiestan por medio de procesos identitarios y representaciones sociales determinadas por la época-espacio en la que se insertan (umbrales). Se pregona que en los contextos urbanos y con el advenimiento de la modernización existiría una tendencia mayor a la lógica individualista, mientras que en el ámbito aldeano-tradicional perviviría mayoritariamente la lógica holista; ambas significan procesos identitarios diferenciados y respuestas específicas con su entorno, que como péndulo van y vienen para influir en los procesos identitarios a lo largo del tiempo. Se podría hablar en términos de identidades comunitarias para contextualizar lógicas predominantemente holistas, y las políticas indigenistas estatales como identificaciones con predominancia de lógica individualista. Con estas abstracciones, en el Cuadro II, se distinguen, dos procesos simbólicos identitarios o mentales según Dumont. 
CUADRO II UMBRALES

(PROCESOS SIMBÓLICOS IDENTITARIOS)

\begin{tabular}{|c|c|}
\hline $\begin{array}{l}\text { COSMOVISIÓN HOLISTA } \\
\text { DE COMUNIDADES }\end{array}$ & $\begin{array}{l}\text { COSMOVISIÓN INDIVIDUALISTA } \\
\text { DE ESTADOS }\end{array}$ \\
\hline $\begin{array}{l}\text { o Continuos renacimientos. No hay fines ni } \\
\text { inicios. } \\
\text { in }{ }^{\circ} \text { Hombre es parte del cosmos junto con la di- } \\
\text { versidad de dioses (polydicea) } \\
{ }^{\circ} \text { Ser humano dentro del mundo (comunalidad) } \\
\text { o Imaginería simbólica de caracteres naturales } \\
\text { (animales, plantas, energía) } \\
\text { o Seres del mundo como entornos. Planteamien- } \\
\text { to integral con el medio ambiente } \\
{ }^{\circ} \text { Humano considerado guardián de la armonía } \\
\text { natural. Interdependiente de lo comunal } \\
{ }^{\circ} \text { Imagen de energías complementarias: vivencia } \\
\text { del presente: regeneración. } \\
{ }^{\circ} \text { Rol de cargos a lo largo del tiempo }\end{array}$ & $\begin{array}{l}{ }^{o} \text { Principio de un inicio y un final } \\
{ }^{o} \text { Hombre en el centro del universo y re- } \\
\text { fleja la centralidad monoteísta de un Dios } \\
\text { (teodicea) } \\
{ }^{o} \text { Ser humano fuera del mundo (misticismo) } \\
{ }^{\circ} \text { Abstracción simbólica del lenguaje teoló- } \\
\text { gico (divinidades sin imagen) } \\
{ }^{o} \text { Ser humano como centro del planteamien- } \\
\text { to moral de su relación con el entorno } \\
{ }^{\circ} \text { Sin posibilidad de diferenciación divina. } \\
\text { Libertad y autonomía del individuo del } \\
\text { grupo. } \\
{ }^{o} \text { Idea de perfección: idea de futuro, bús- } \\
\text { queda de eternidad } \\
{ }^{\circ} \text { Cargo jerárquico y único perenne. }\end{array}$ \\
\hline
\end{tabular}

Fuente: Elaboración propia

De ahí que se pueda esquematizar que las políticas públicas de Estado son productos en la larga duración de cosmovisiones especificas, en este caso la individualista representadas por las instituciones modernas de poder, que a su vez, entrechocan con dinámicas de pertenencia comunitarias que responden a herencias históricas de lógicas holistas, lo que termina por traducirse en conformaciones de identidad como el autoadscribirse como "indígenas".Los adjetivos identitarios nos dan cuenta de las relaciones asimétricas de poder, las referidas a los "indígenas" son más visibles analíticamente. $\mathrm{Si}$ algún miembro de pueblos nacionales viste atuendos y trajes tradicionales para recibir de funcionarios públicos algún tipo de ayuda, se dice que esa persona se "disfraza de indígena" para poder obtener recursos estatales. ¿No es acaso similar situación cuando un "urbano" se disfraza con traje sastre y corbata para una entrevista de trabajo en la ciudad?

Las representaciones en cuestión nos permiten entender las diversas respuestas que los involucrados logran movilizar, desde sus diversos elementos identitarios contenidos en su haber a lo largo de sus experiencias personal y grupal, así como, la manera en la cual aprehenden y transforman dichas políticas en su cotidiano, con consecuencias benéficas o perjudiciales para ellos. La interacción de funcionarios de Estado con los miembros de las comunidades definen las diferentes posturas, acciones y modos que los grupos de sociedades comunitarias han adoptado a lo largo del tiempo, al punto de conformar identificaciones momentáneas (hibridación/ re-significación) con insumos identitarios permanentes (sentidos de pertenencia). Se podrían comparar con otras regiones del mundo donde la relación Estados y sociedades pluri-étnicas son igualmente sustantivas (Bolivia, Ecuador, Guatemala, Chile, Brasil, Paraguay, entre otros). Las cosmovisiones (holistas/ individualistas) se expresan teóricamente a través de identidades, y traducen en su modo más concreto, formas de organización, acciones colectivas, transformación del entorno. Una acción colectiva puede ser desde una política pública estatal, hasta estrategias grupales que se heredan y/ o actualizan, a través de la tradición o por medio de la reacción al entorno en el que se vive. 


\section{Identidades comunitarias y holismo/ políticas indigenistas e individualismo7}

Con todas las precauciones que esto conlleva, el planteamiento teórico-analítico aquí esbozado, puede ser ejemplificado con la relación que existe entre comunidades nativas e instituciones estatales; entre acciones de la comunidad local y políticas públicas; entre familias cercanas y funcionarios públicos. Cada una de estas entidades contienen, expresan y favorecen formas de pensar, creer y racionalidades específicas (cosmovisiones) nutridas por la memoria colectiva y actualizadas con los diversos modos de accionar, reaccionar a las diversas interacciones y entornos implicados. Estas, interrelación e influencia, se han buscado visibilizar, a partir de casos concretos ${ }^{8}$ con las comunidades llamadas indígenas por el estado mexicano, que como es ya sabido son diferenciadas entre sí, compartiendo núcleos duros comunes de larga duración que hemos denominado holistas. ${ }^{9}$ Se trata de dos formas de sentimientos de pertenencia, que esquemáticamente, nos permiten visibilizar formas opuestas de organizar e interpretar los entornos, pero que a su vez se influyen mutuamente. ${ }^{10} \mathrm{Para}$ el caso de México los llamados indígenas se identifican por narrativas de exclusión, de marginalización, de paternalismo, y en su defecto de re-consideración, de resistencia étnica cultural. Comparten su historia de estigmatización y más reciente de discriminación positiva, que a lo largo del tiempo se les ha imputado, al punto de formar significados de pertenencia comunes, lo cual no significa que sean iguales culturalmente hablando. Serían indígenas para el Estado, en términos identitarios, sólo por que comparten los atentados a su cultura que las relaciones de poder hegemónicas les han propinado a lo largo del tiempo. Ello alimenta la polaridad de características: identitarias comunitarias versus características identitarias indigenistas (Cuadro Umbrales III).

7. Son aquellas políticas públicas de corte nacional, que en los últimos años se han planteado como la panacea al problema de la pobreza y marginación, y cuyo objetivo es la movilidad social, las cuales han sido llamadas, para el caso de México como Solidaridad, Progresa, Contigo, Oportunidades, Enlace, etc., que no son más que políticas vertientes del Banco Mundial y el PNUD, "tropicalizadas" en México, donde según el nuevo gobierno al frente, las denominaciones pueden cambiar. Desde los años ochentas, se llevan a cabo estas políticas en México, cuyo objetivo es que a través de la dádiva del gobierno de una mensualidad de 100 dólares en promedio, (a las familias, consideradas más pobres del país), se genere movilidad social en las próximas generaciones. A cambio, las familias se comprometen a llevar a sus hijos a la escuela y las mujeres a ir al médico cada mes. A una gran mayoría de pueblos llamados "indígenas" le son aplicados estos programas, convirtiéndose en realidad en "políticas indigenistas".

8. Tanto la política del catecismo de ayer como las políticas multiculturales de hoy no difieren en cuanto a su consideración esencialista de los "indígenas", así como en cuanto a la lógica de racionalidad individualista.

9. Para solo mencionar algunos ejemplos al respecto; comunidades como las mexicas, otomíes, purépechas, mixtecas, araucanos, zapotecas, nahuas, mayas..., además de ser diferentes entre ellas al nivel supra-étnico, están diferenciadas al nivel intra-étnico; desde el lenguaje, rituales y modos de presentación. Dicha diferenciación puede llegar a ser de tal magnitud de no comprenderse idiomáticamente de manera clara entre ellas, tal y como se puede observar con el árabe, donde se presentan diferenciaciones entre la cultura árabe-libanesa, y la del Egipto central, que a pesar de compartir fondos simbólicos comunes (cosmovisiones), cada una resguarda sus diferencias en formas. Aquí es donde podemos entender la diferencia conceptual entre Identidades (fondos simbólicos compartidos) e Identificaciones (presentaciones diversificadas).

10. Se aclara que aquí no se busca hacer de un caso etnográfico una generalidad antropológica; ni decir que todos los grupos otomíes o "indígenas" actúan homogéneamente y son iguales; menos aún, que se trata de dinámicas identitarias específicas de algunos grupos particulares denominados por algún adjetivo impuesto. 


\section{CUADRO UMBRALES III}

(CARACTERÍSTICAS IDENTITARIAS)

\section{PRINCIPIOS COMUNITARIOS}

${ }^{\circ}$ Pertenencia al barrio y comunidad.

${ }^{\circ}$ Relación con su espacio de manera colectiva. En la "casa de la comunidad" las actividades son afuera con su entorno y los demás, en el espacio público

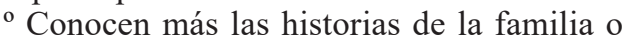
vinculadas con la comunidad que la historia universal. La comunidad es el "ombligo del mundo". Es la historia local.

${ }^{\circ}$ No hay un gran interés por cultivar tradiciones de otros lados. Interrelación con el campo, la naturaleza y el entorno.

${ }^{\circ}$ Dificultades para actuar en términos de competencia. Puede haber "envidias" pero no competencia uno contra el otro.

${ }^{\circ}$ Se elogia la pertenencia de grupo, con sus particularidades y sus procesos de diferenciación frente a otras comunidades, reforzando la identidad comunitaria.

${ }^{\circ}$ La comunidad es un ente cultural intrínseco y un agente social frente al Estado. Las competencias sociales se dan en el seno de la familia.

${ }^{\circ}$ Familia es el espacio de toma de decisiones, establecimiento de valores morales y socialización de los modelos culturales.

${ }^{\circ}$ La identidad se define con el entorno.

\section{PRINCIPIOS INDIGENISTAS}

'Sentido de pertenencia a sí mismo.

${ }^{\circ}$ El espacio se refiere a un YO, y las actividades de socialización son adentro de la casa, en el espacio privado.

- Se requiere de una inercia en la planificación y estrategia. La universalidad es el centro del mundo. Referentes a la historia universal y occidental, y no a la local.

${ }^{\circ}$ Las políticas buscan destacar los contenidos de expresión de las culturas "indígenas", pero en un marco nacional-homogéneo, ecencialista y no comunitario.

${ }^{\circ}$ Valores cimentados en la urbanidad, el civilizacionismo, la competencia entre individuos y "racionalidad instrumental".

${ }^{\circ}$ El discurso está construido en torno al progreso de la civilización, funcionalizando la pertenencia a la Nación, a una identidad única e indeleble.

${ }^{\circ}$ La diversidad como proceso de integración global de valores impuestos como universales, refuncionalizando el localismo culturalista (las lenguas maternas locales sólo fungen como elementos de diferenciación globalizada).

o Transmisión de valores con principios de socialización y aprendizaje para la toma de decisiones individuales.

${ }^{\circ}$ Defino el mundo con respecto al Ego.

Fuente: Elaboración propia ${ }^{11}$

En suma, las políticas públicas en general, y las indigenistas en particular, se ven nutridas de racionalismo secular individualista, de modo que en sus expectativas de respuestas, de resultados y de comportamientos se esperan reacciones de los involucrados, con sustratos principalmente individualistas, a los cuales muchos miembros de comunidades no responden de la forma esperada. ${ }^{12}$ Por su parte, la lógica holista se ve enriquecida con formas de pensar llamadas colectivas, véase tradicionales (comunitarie$d a d$, racionalidad colaborativa...), cuyos pueblos en cuestión hacen que las reacciones,

11. Elaboración con base en trabajo de campo en comunidades hñahñus del Estado de Querétaro, Hidalgo y Estado de México 2007 y 2016, así como con comparaciones monográficas consultadas referentes a otros pueblos en el continente americano (Millán Saúl/ Julieta Valle, 2003: 143-207).

12. Las políticas públicas, particularmente las indigenistas, terminan por ejercer, a través de su imposición, identidades que podemos llamar híbridas y de resignificación que se conforman en la interacción cotidiana entre Estado y poblaciones nativas a lo largo de los tiempos, al punto de crearse identidades incuestionables por los mismos grupos a los que se les ejerce tal imposición. 
las expectativas, la racionalidad del mundo, tiendan principalmente a caracterizarse por un proceso de pensamiento holista, es decir colectivo, para el cual las políticas de Estado aplicadas sobre pueblos y territorios, a lo largo del tiempo por la institución hegemónica (Iglesia, Reino, República, Estado-Nación), son casi de lógica opuesta. Las repercusiones de estos choques, que terminan siendo identitarios, tienen que ver con fenómenos de exclusión, marginación, pobreza, o en todo caso subdesarrollo que se generan entre poblaciones al interior de los Estados. Para nuestro caso, las políticas indigenistas reproducen principalmente la desigualdad social en el seno de las comunidades y pueblos nativos.

En el interior de las comunidades hay diferencias y graduaciones de identidades entre los miembros que vale la pena tener presentes. Cada uno de los pueblos y comunidades construyen sus identidades con base, ciertamente a un referente y creencias comunes, pero con matices propios de cada quien, los cuales en muchos casos están directamente relacionados con el impacto que las políticas públicas han ejercido sobre las estrategias históricas de pertenencia. Por ejemplo el impacto de las políticas indigenistas en las poblaciones yaquis, del norte de México, ha sido menos intenso, por lo cual los sentimientos de pertenencia a la comunidad han sido más fuertes y sólidos, mientras que las políticas indigenistas en las regiones otomíes han sido más presentes e intrusivas, lo que ha provocado que el sentimiento de pertenencia sea más diluido, al punto que los niños y los pobladores hablen menos sus idiomas, los valoren menos, así como los saberes y formas de hacer y pensar de la comunidad. ${ }^{13} \mathrm{En}$ efecto, tal como sucede también con el proceso de identificación de los sistemas políticos del Estado, en los pueblos la pertenencia identitaria a la comunidad mantiene valores específicos a ella, que se transmiten en cada generación. Esta transmisión se lleva a cabo en todo proceso identitario a través de construcciones discursivas, en su sentido más amplio (visual, lingüística, simbólica), que a su vez están precedidas y basamentadas por uno o varios sistemas de creencias, que son legítimos y verdaderos porque se cree en ellos. Para el caso específico de nuestra investigación, ejemplificamos esta reflexión, a partir de trabajos de campo realizados en la última década. ${ }^{14}$

\section{Ontogénesis en las identidades comunitarias}

Los miembros de las comunidades tienen que lidiar al mismo tiempo al menos con dos procesos identitarios diferenciados, cuando se imponen las políticas públicas en su territorio: el sentimiento de pertenencia que se encuentra en sus grupos, sus familias y comunidad; y el sentido de pertenencia que se ubica, en este caso, con la identificación del sistema político estatal. Es a partir del estudio de la relación entre cosmovisiones intrínsecas en las políticas públicas indigenistas de Estado (individualistas) y en las

13. Recientemente con el reavivamiento de la diversidad y el discurso políticamente correcto de los "pueblos indígenas", la revaloración de la lengua, costumbres, cosmovisiones vienen a la alza, como lo demuestra los innumerables festivales culturales que en nombre de los "pueblos indígenas" se realizan cada vez más recurrentemente por diferentes instituciones y poblaciones en general.

14. El trabajo de campo se llevó a cabo en las comunidades hñahñus u otomíes de los estados de Querétaro en los municipios de Tolimán y Casablanca, en el Estado de Hidalgo en el valle del Mezquital, y en el municipio de Temoaya en el estado de México, durante los años de 2007 y el 2015. Se realizaron 1800 entrevistas semiestructuradas a pobladores de grupos etarios de entre 20 a 60 años de edad. Las preguntas tuvieron que ver sobre la percepción con respecto a las políticas públicas de combate a la pobreza de corte liberal aplicadas a la comunidad, así como políticas asociadas a la educación básica bilingüe (EBB). Se preguntó sobre percepción con respecto a la presencia de la Iglesia en la educación estatal (protestantes y católicas) en las comunidades, y finalmente la importancia de sus tradiciones y ritos comunitarios de corte ancestral. 
lógicas comunitarias (holistas) que se pueden visibilizar los procesos identitarios de hibridación/ resignificación, lo que se traduce por el conflicto entre la experiencia dóxica familiar, la experiencia identitaria con cada comunidad de origen (que es la más perdurable) y la experiencia (doxa) con la institución estatal, que con frecuencia se vincula con la prolongación de una experiencia que se distancia del mundo vivido en su comunidad, generando al final de cuentas la reproducción de la desigualdad social. ${ }^{15}$

En un estudio publicado sobre las representaciones,- aceptación, percepción y reacción- que se tenía en una comunidad otomí de las políticas públicas de Estado de combate a la pobreza (Progresa-Oportunidades), los autores García y Valencia (2003), -sin denominarlo así -dieron cuenta de procesos identitarios ahí involucrados.

El punto nodal aquí, es la importancia de comprender los procesos identitarios en el accionar diario, que se ponen en juego en la relación comunidades y políticas públicas donde se entremezcla el balanceo entre identidades comunitarias e identidades indigenistas, las cuales van interactuando según el contexto y el momento, fungiendo como estrategias de negociación, supervivencia para las poblaciones concernidas, ${ }^{16}$ que unos llamarían resignificación (Martínez-Casas, 2001), y otros hibridación (Canclini, 1995).

\section{III) Procesos identitarios y sentidos de pertenencia: hibridación o resignificación}

En este sentido, ¿de qué modo acciones y estrategias identitarias de hibridación y resignificación, los integrantes de pueblos y comunidades nativos en México han llevado a cabo, en reacción a las implementaciones de políticas indigenistas estatales? En las comunidades como en los pueblos y barrios, cada uno de sus miembros tiene y mantiene un rol específico que actúa independientemente o en interdependencia con los demás, y funcionan como formas de estrategia y negociación con su entorno (propuesta goffmaniana de la multiplicidad de roles y máscaras que de actor social). Los miembros de las comunidades, optimizan su relación con el entorno, y particularmente con las políticas de Estado, con al menos dos lógicas que aquí denominaremos de hibridación y de resignificación. Este ángulo trata efectivamente de romper con el hito de la identidad

15. Los factores económicos y sociales juegan un papel preponderante con el poco éxito obtenido en las comunidades con dichas políticas. Según la encuesta del INEGI de 1995, para una población de 229350 de hablantes de lengua indígena de 6 a 14 años, 33\% no sabe leer ni escribir. Véase igualmente (Bravo Ahuja 1977). Los datos no cambian para el censo del INEGI, 2000 y 2010, empero los procesos mentales inscritos en dichos programas infieren de manera negativa en los aspectos identitarios de los actores que ahí se ven implicados. Las políticas indigenistas generan más desigualdad social que lo que buscan erradicar porque se siguen estableciendo con base en la imposición de una racionalidad específica y la esperanza de que finalmente los concernidos se adhieran a ellas y actúen con tal racionalidad.

16. En otro ejercicio de auto-percepción realizado en los talleres propedéuticos para mujeres indígenas en 2016, con el fin de ingresar a las maestrías de excelencia nacionales, convocadas por parte del Consejo Nacional de Ciencia y Tecnología de México sede Guerrero, se les preguntó a las convocadas lo que significaba ser para ellas "mujer indígena". Las repuestas se aglutinaron en torno a considerarse pobres, con una lengua diferente al español, con una cultura local, a veces milenaria, "pero eso sí con mucha dignidad". No se necesita hacer una encuesta nacional representativa para validar estos datos cualitativos, cuando basta con escuchar diariamente las mismas percepciones vertidas a los funcionarios públicos de Estado por ciertos miembros de las comunidades, particularmente cuando se trata de obtener beneficios económicos (becas, programas sociales, premios, leyes y derechos etc.). Promovidas por organismos, que para el caso de México, son muchas y con una larga tradición institucional (INHALI, CDI, INAH, etc.). Por lo contrario, si se hace la misma pregunta a personas que no se catalogan como "indígenas", que no se auto-perciben así, que no hablan alguna lengua nacional materna, por ende tampoco se sienten en una condición de pobreza, entonces más bien se expresan en términos de considerarse desempleadas o "sin dinero". Pobreza y cultura ancestral no se mezclan ni mencionan en estas respuestas. 
comunitaria enclaustrada en dos polos fijos (identidad indígena, identidad étnica), a veces opuestos, a veces complementarios, al punto que se hable en ocasiones de "identidades sincréticas". No se pueden negar por completo estos procesos subjetivos, pero las identidades se van construyendo, re-estructurando y complejizando en las comunidades a lo largo de la historia a partir de la relación que éstas han mantenido con las políticas públicas del Estado. Se trata de procesos de identificación (movibles, cambiables...), sentidos de pertenencia, insertos en proceso identitarios más estructurados, sentimientos de pertenencia (cosmovisiones). Por ello se postula que contrariamente a la definición de identidades sincréticas, mestizas, populares, étnicas, que se han venido postulando en los últimos años para esquematizar formas de comportamiento yacientes en las llamadas comunidades "indígenas", existen otros procesos identitarios de hibridación y resignificación, que dan cuenta de comportamientos estratégicos, de supervivencia, de resistencia y de creatividad en pueblos y comunidades, sean éstas criollas, urbanas, o como se las denomine. Se juegan diversos esquemas identitarios a la vez, revelan las relaciones de poder existentes, de larga data, entre poder hegemónico y pueblos. Se habla así de hibridación o de resignificación en cualquier contexto urbano o rural, al no ser más que estrategias vinculadas con la construcción cultural del sentido de pertenencia. Son modos de adaptación a las diferentes situaciones en las que las personas o grupos interactúan en la ciudad o en el campo con las políticas públicas (en este caso las indigenistas).

Para Martínez-Casas (2001) la resignificación es "el proceso complejo, tenso y conflictivo que presentan los migrantes para experimentar su cultura indígena, campesina y corporativizada, tanto en el contexto urbano como en su comunidad de origen con la cual mantienen fuertes vínculos materiales y simbólicos. Resignificar no implica un cambio cultural (aculturación) sino la adquisición de un conjunto de competencias sociales que amplían el espectro de significaciones posibles de la cultura indígena en el medio urbano y en las relaciones rurales en función de los contextos interactivos en los que los migrantes se mueven. [...] La resignificación implica [... en condiciones de contacto con grupos urbanos...] la coexistencia de modelos culturales distintos en el contacto entre grupos que no se aculturan, sino que mantienen patrones e identidades indigenas en contextos no-indigenas". ${ }^{17}$ Para el caso que nos concierne consideramos que la representación de los actores tiene un rol mucho más importante que la interpretación que puedan hacer de los diferentes habitus (Bourdieu, 1994) con los que se enfrentan. Los agentes sociales, en este caso los miembros de las comunidades, han aprendido a lo largo de la historia a responder frente a las imposiciones, invasiones o convivencias con el Otro, han aprendido a movilizar sus estrategias y a utilizar diferentes atuendos de respuestas y máscaras de resistencias frente a su entorno, principalmente frente al Cura, el Maestro, el Funcionario de gobierno, al Médico, ${ }^{18}$ todos ellos representando una Institución perteneciente al Estado-nación y por tanto vehiculando un sistema de valores y creencias específicos. Se trata por tanto de movilizaciones que no impiden por supuesto que al mismo tiempo se vayan reestructurando sus formas de vivencia, o

17. "La resignificación tampoco implica asimilación en los términos más en boga en la antropología actual que analizan la progresiva acomodación lingüistica y cultural de los migrantes. Es una propuesta diferente que pretende dar cuenta de la oscilación cultural presente en los migrantes entre los valores y patrones de su comunidad indigena y los de la vida urbana, en función de los diferentes contextos interactivos en los cuales deben aprender a moverse" (Martínez-Casas 2001:7-8)

18. Se trata por supuesto de ideales tipo, que simbólicamente pueden ser usados como tipos-ideales en el sentido del poder de legitimación que transmiten. 
supervivencia, jugando entre una y otra experiencias vividas que se presentan. Son los sentimientos de pertenecía que se resignifican a partir del entorno e interacción vividos.

Lo anterior no deja de contener varias consecuencias en cuanto al proceso de lo que llamamos aquí "hibridación de la identidad", es decir, la separación de diferentes estrategias de comportamiento frente a un grupo de pertenencia constituida a través de la experiencia vivencial. Estas estrategias se pueden asumir de manera consciente, pero esto no deja por tanto de presentar características de desfragmentación de los campos identitarios. Estos campos se pueden reforzar (resignificación) pero también debilitar o resquebrajar (hibridación). En suma, la definición que hacemos aquí de hibridación, tiene que ver con los procesos orgánicos identitarios que no llegan a permear por completo los procesos mecánicos identitarios, mientras que la resignificación (MartínezCasas 2001) es un proceso identitario que llegar a constituirse de manera integral en la interacción diaria. Cabe insistir que el proceso de hibridación aquí expuesto, está fuertemente relacionado con un contexto comunitario y en relación (percepción y recepción) con las políticas públicas establecidas por el Estado-nacional. La hibridación, es un proceso de integración mucho más lento que el llevado a cabo a través de la resignificación, pero es igual de persistente y presente. En suma, la resignificación se lleva a cabo la mayor parte de las veces, cuando no existe de inicio una estructura de pertenencia de origen, que lleva a que las constituciones identitarias de las comunidades en su relación con el entorno tienda a resignificarse, mientras que en un contexto en donde existe una estructura de pertenencia de grupo sólida, el proceso de constitución de identidad con respecto al entorno es de hibridación (de apropiación de roles según el contexto en el que se interactúe, esto es un proceso de identificación).En fin, el proceso de resignificación da cuenta de un comportamiento que está inserto en una dinámica descontextualizada del mundo comunitario o tradicional, donde las políticas públicas no están forzosamente pensadas para los miembros de la comunidad, por lo que su relación con las políticas públicas es diferente que en una contextualización comunitaria tradicional, lo que lleva a que se favorezca, en contexto de modernización, más la resignificación que la hibridación.

En este sentido, la paradoja es que en la relación entre diferentes sistemas de creencias en la comunidad de origen (proceso de hibridación identitaria) el proceso identitario es más violento (marginación, reproducción de la pobreza, agresión intrafamiliar...) que el encontrado en un contexto de resignificación (contexto modernista la mayor parte del tiempo). Pues en este último tal y como se ha planteado con respecto a la ciudad y la identidad, es más factible que en las ciudades sobre todo las grandes ciudades, se facilite el desarrollo de una lógica de anonimato, y la identidad pueda encontrar nichos íntimos de protección. Mientras que en el contexto comunitario, el ataque o la relación es mucho más directa, el Otro (el Estado con sus políticas públicas) entra por "la parte de atrás de la casa". Existe por tanto menor factibilidad de refugio. De tal modo que la respuesta frente a otros modos de pensamiento es diferente que el encontrado en un contexto de modernización (resignificación). De ahí se puede establecer la diferencia de respuestas que puedan existir entre la llamada resignificación (proceso identitario), y la hibridación (proceso de identificación). Las dos formas existen pero, dependiendo de los contextos, pueden tender a ser más predominantes uno que otro proceso, particularmente cuando se habla del impacto de las políticas públicas del Estado-nación en los sentidos/ sentimientos de pertenencia en contexto comunitario (hibridación/ proceso de identificación). Así, se podrá plantear en esta problemática específica que la respuesta identitaria en el contexto de la comunidad se encuentra en la lógica del 
actor social; mientras que en un contexto de modernización se encuentra en la lógica del agente social. En el primero hay una lógica de Representación, en la segundo de Interpretación.

\section{Hibridación (pausamiento) y Resignificación (inmediatez)}

¿Podríamos decir que en el contexto de la comunidad de origen encontramos una lógica identitaria de resignificación constante (en el marco de un proceso de hibridación), mientras que en el contexto de un imaginario de la comunidad de origen (modernización) encontramos una lógica identitaria de hibridación constante (en el marco de un proceso de resignificación)? Estas dos lógicas funcionan principalmente en circunstancias de relaciones diferenciadas, en reacción con el Otro diferenciado. Las dos estrategias son de protección y resguardo de la identidad o comunidad de origen, es decir, del mítico identitario (identidad comunitaria). La hibridación sería entonces un proceso de absorción/dominación, mientras que la resignificación estaría inscrita en un proceso de resistencia/repulsión. Somos por tanto actores sociales. Sin duda este elemento nos permite a la postre re-significar en términos de estrategia nuestro sentido de pertenencia identitaria. Existe un proceso de ida-y-vuelta. La tarea para el científico social es detectar cuándo ocurren estos procesos.

\section{Identidades comunitarias y políticas indigenistas: a manera de conclusión}

42 Lo anterior no sólo habla de un esquema de comprensión de procesos identitarios sino que nos advierte que las políticas indigenistas más que dar respuesta a la diferenciación de mundos de vida, produce y genera más violencia en el seno mismo de las comunidades, o en su caso más desigualdad social que la que busca erradicar con sus políticas. Desde nuestra perspectiva la Resignificación plantea una disyuntiva: si bien la resignificación significa una movilización de estrategias, - apropiarse de elementos diferenciados, lógicas de respuestas e incluso de resistencia -, esta resignificación difícilmente se presenta lo suficientemente legítima como para lograr posicionarse frente a las políticas públicas, resultado de un sistema de creencias específico aún dominante y hegemónico.

Si la resignificación implica posibilidades de extensión económica, véase política, éstas deben por tanto fungir como potencialidades concretas para una concreción del ideal de bienestar manifiesto en los miembros del grupo. Desafortunadamente parece que éste no es el caso y aún se siguen encontrando situaciones de inequidad en las comunidades nativas dentro del mismo proyecto democrático del Estado-nación. Por tanto esta resignificación (construcción de herramientas de respuesta a través de la reapropiación de elementos exógenos a su sustento identitario) solo podrá tomar cuerpo y validez concreta si se logra potenciar dichas lógicas. Este es sin duda el desafío del sistema de valores y de creencias dominante vinculados en la relación pueblos-Estados nacionales, (al cual se le debe quitar toda la connotación negativa de tipo dialéctica que ha venido heredando) que se ve reflejado en el proyecto democrático multicultural contemporáneo. Hay quienes optarán por una política del affirmative action como primera estrategia de reforzamiento de alternativas políticas en cuestión de la identidad (que no deja de estar alejada de las políticas estilo Progresa multiculturales, aunque con sus matices cualitativos). Si bien no se desdeña estas propuestas, es indispensa- 
ble enriquecerlas con un aporte vinculado a las creencias y la interacción que de ello procede, es decir, con aquello que ofrece parámetros de vida, de comportamiento y de convivencia con el entorno social y cultural. Esto sin duda es posible integrando en el análisis lo que Weber llamaría el sentido de la acción, es decir, el sentido que se da a lo cotidiano, a los comportamientos y a la alteridad, significados sociales que se generan colectivamente en la interacción diaria. Esto se refiere sin duda alguna al innegable proceso social del sentimiento de pertenencia presente en toda interacción social.

Este sentido de pertenencia reconforta la pasión común, participa de un rito de anamnesis cotidiano, en una palabra celebra el clan al que se pertenece: sentimiento de pertenencia. Ciertamente se trata de una expresión más o menos brutal del sentimiento de pertenencia tribal, comunal, afectivo, que termina por recobrar una fuerza viva, bárbara, que el discurso de la Modernidad, del Estado-nación, y de la políticas desarrollistas, creían haber superado, y que se impone en la actualidad con virulencia en la retórica que el cuerpo social tiene sobre sí mismo. Identidades comunitarias remite entonces a cualquier proceso identitario de base que conforma sentires (sentimientos/ sentidos) de pertenencia en cualquier grupo o persona, con la característica de ser identidades reforzadas por la comunidad, a partir de los insumos externos identitarios que se les imponen y predominan. Nunca se es más "indígena" que cuando hay una imposición de políticas en la comunidad de pertenencia. En este sentido nunca hemos sido todos más "indígenas/ indios" que ahora.

\section{Bibliografía}

Aguirre Beltrán, Gonzalo, 2013 El proceso de aculturación. México, FCE. [1957]

Anderson, Benedict, 1993, Comunidades Imaginadas, reflexiones sobre el origen y la difusión del nacionalismo. Mexico: FCE, Col. Popular.

Bartolomé, Miguel y Barabas, Alicia (coord.), 1988, Autonomías Étnicas y Estados Nacionales. México: CONACULTA/INAH.

Bohórquez Molina José Gerardo, Alberto García Espejel, Diego Prieto Hernández, Marco Antonio Rodríguez Espinosa, Los Pobres del Campo queretano. Política Social y combate a la pobreza en el medio rural de Querétaro, INAH, México, 2003.

Bourdieu, P., 1994 :129, Raisons Pratiques, sur la théorie de l'action, Paris, Seuil.

Bravo Ahuja, G., 1977, La enseñanza del español a los indígenas mexicanos, los materiales didácticos, México, El Colegio de México.

Broda, Johanna y Báez-Jorge, Félix (coordinadores), 2001, Cosmovisión, Ritual e identidad de los pueblos indígenas de México, México: CA, FCE.

Dumezil, G., 1941, Jupiter, Mars, Quirinus, París, Gallimard.

Dumont, L. 1966, Homo hierarchicus, le système des castes et ses implications, Paris:Gallimard.

Durand, G., 1980, L'Ame tigrée, lês pluriels de psyché. París, Denoël-Médiation.

, 1984, La Foi du Cordonnier, París, Denoël.

Durkheim, Emile, 1930, De la division du travail social. Paris, PUF.

Freund, J., 1983, Sociologie du conflit, París, PUF.

Galinier, Jacques, 1990, La mitad del mundo. Cuerpo y cosmos en los rituales otomíes. México, UNAM

García Canclini,Nestor, 1990, Culturas híbridas: estrategias para entrar y salir de la Modernidad, México, Grijalbo. 
García Falcón, Sulima y Valencia Lomelí, Enrique, 2003:95-102, “Las representaciones en torno al Progresa-Oportunidades en Santiago Mextititlán”, Guadalajara, Revista Universidad de Guadalajara, Número 27.

Geertz, Cliford, 1987, La interpretación de las culturas. México, Gedisa.

Habermas, J., 1990, Teoría de la acción comunicativa. Buenos Aires, Taurus.

López, Austin, 1999:120, Breve historia de la tradición religiosa mesoamericana. Mexico, Colec. Textos Serie Antropología e Historia Antigua, UNAM, Instituto de Investigaciones Antropológicas.

Maffesoli, Michel, 2007, En lo profundo de las apariencias, por una ética de la estética, México, Siglo XXI editores. ,2005 La transfiguración de lo político, México, Herder. ,2004, El Tiempo de las tribus. El ocaso del individualismo en las sociedades posmodernas, México, Siglo XXI editores.

,2004,La tajada del diablo, Compendio de subversión posmoderna, México,Siglo XXI editores.

\section{Económica.}

,2004, Del Nomadismo, Vagabundeos iniciáticos, México, Fondo de Cultura

Martínez-Casas, R, 2001, Una cara indígena de Guadalajara: la resignificación de la cultura otomí en la ciudad, Tesis de Doctorado en Ciencias Antropológicas, México,UAMIztapalapa.

Martuccelli, D., 2002, Grammaires de l'individu, Paris, Fol essais, Gallimard.

Millán, S./ Julieta Valle (Coordinadores), La Comunidad sin límites. Estructura social y organización comunitaria en las regiones indígenas de México, Vol. II, Etnografía de los pueblos indígenas de México, INAH, México, 2003, pp. 143-207.

Rist, Gilbert, Le développement: Histoire d'une croyance occidentale, Presses de Sciences Politiques, París, 1996. 